\title{
Upstream signalling of mTORC1 and its hyperactivation in type 2 diabetes (T2D)
}

\author{
Muhammad Ali, ${ }^{1, *}$, Shazia Anwer Bukhari ${ }^{1}$, Muhammad Ali ${ }^{2} \mathcal{E}$ Han-Woong Lee ${ }^{3, *}$ \\ Departments of ${ }^{1}$ Biochemistry, ${ }^{2}$ Zoology, Government College University, Faisalabad, 38000 Pakistan, ${ }^{3}$ Department of Biochemistry, \\ College of Life Science and Biotechnology, Yonsei University, Seoul 03722, Republic of Korea
}

\begin{abstract}
Mammalian target of rapamycin complex 1 (mTORC1) plays a major role in cell growth, proliferation, polarity, differentiation, development, and controls transitioning between anabolic and catabolic states of the cell. It collects almost all extracellular and intracellular signals from growth factors, nutrients, and maintains cellular homeostasis, and is involved in several pathological conditions including, neurodegeneration, Type 2 diabetes (T2D), obesity, and cancer. In this review, we summarize current knowledge of upstream signaling of mTORC1 to explain etiology of T2D and hypertriglyceridemia, in which state, the role of telomere attrition is explained. We discuss if chronic inhibition of mTORC1 can reverse adverse effects resulting from hyperactivation. In conclusion, we suggest the regulatory roles of telomerase (TERT) and hexokinase II (HKII) on mTORC1 as possible remedies to treat hyperactivation. The former inhibits mTORC1 under nutrientrich while the latter under starved condition. We provide an idea of TOS (TOR signaling) motifs that can be used for regulation of mTORC1. [BMB Reports 2017; 50(12): 601-609]
\end{abstract}

\section{INTRODUCTION}

The mammalian target of rapamycin (mTOR), is conserved in all eukaryotes including plants, worms, flies, and mammals (1). mTOR comprises two complexes, mTORC1 and mTORC2, among which mTORC1 is directly regulated by nutrient status of the cell. mTORC2 is indirectly regulated by RTK and activation occurs after activation of mTORC1 $(2,3)$. Mammalian mTORC1 is mainly composed of mTOR, Raptor, and G $\beta$ L; the complex serves as a staple hub for upstream signaling (4).

*Corresponding authors. Han-Woong Lee, Tel: +82-2-2123-5698; Fax: +82-2-2123-8682; E-mail: hwl@yonsei.ac.kr; Muhammad Ali, Tel: +92-41-9200037; Fax: +92-41-9201416; E-mail: mali@gcuf. edu.pk; m.ali@yonsei.ac.kr

https://doi.org/10.5483/BMBRep.2017.50.12.206

Received 26 October 2017

Keywords: Diabetes, Hypertriglyceridemia, Insulin resistance, mTORC1 restriction
Nutrient-rich conditions facilitate translocation of mTORC1 to the lysosome, and thus regulate activation of this complex (5, 6). This complex receives intracellular and extracellular signals and controls cell size, growth, and proliferation by performing the anabolic function of protein synthesis, lipid synthesis, and mitochondrial metabolism (7-9).

mTORC1 activity is regulated by a plethora of upstream signaling elements. It collects upstream signals from growth factors, stress, energy, oxygen, glucose, and amino acids and promotes synthesis of proteins, lipogenesis, lysosome biogenesis, and activates energy metabolism $(7,8,10)$. Newly discovered elements TERT and HKII inhibit mTORC1 activity under amino acid and glucose starvation, respectively (Fig. 1) $(11,12)$.

Diabetes is associated with obesity linked with prolonged intake of high energy diet. Dietary proteins generate circulating amino acids that activate the mTORC1 (13-15). The condition causes mTORC1 hyperactivation that over a prolonged period leads to insulin resistance, hypertriglyceridemia, and hyperlipidemia (16).

Loss of chromosomal DNA by telomere attrition has deleterious effect on numerous cellular functions. Telomere shortening leads to DNA damage, cellular senescence, and apoptosis that is linked with aging disorders (17). Short telomeres play an important role in pathogenesis and disease progression of T2D. Short telomeres increase probability of beta-cell senescence, reduce insulin secretion, and exhibit mitochondrial dysfunction $(18,19)$.

The purpose of this review is to discuss signaling molecules and external factors that affect mTORC1 regulation. We summarize current knowledge of how mTORC1 hyperactivation leads to T2D diabetes and hypertriglyceridemia. We added the role of telomerase attrition in T2D. Finally, we discussed a possibility if pharmacological inhibition of mTORC1 can reverse incidence of T2D. We suggested that inhibition of mTORC1 as well as restriction of mTORC1 may be useful in treating hyperactivation of this complex in T2D.

\section{UPSTREAM REGULATORS OF MTOR COMPLEX 1}

Amino acids

Amino acids regulate mTORC1 through different signaling elements. Among amino acids, leucine plays an important role 


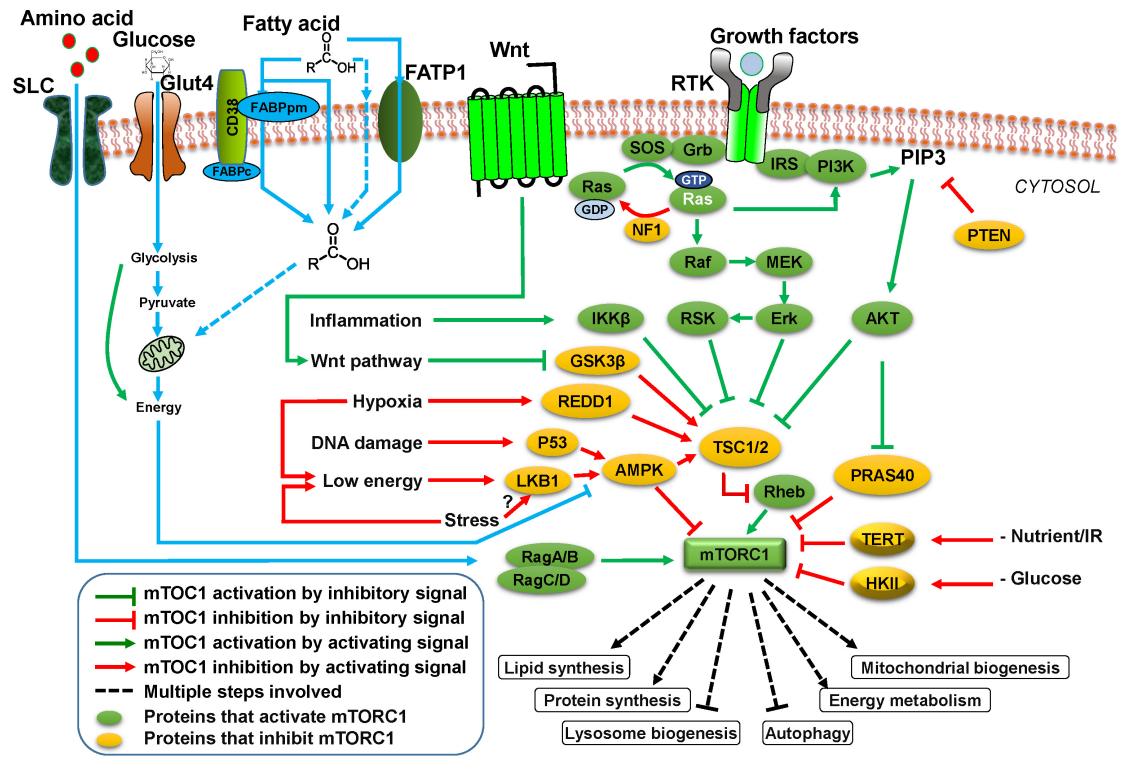

Fig. 1. mTOR signaling pathway and its upstream elements. Amino acids, glucose, fatty acids, Wnt ligand, growth factors, hormones, stresses, energy status, and oxygen are key factors that affect mTORC1 activity. Upon activation, mTORC1 facilitates the synthesis of protein, lipids, and energy metabolism. It inhibits autophagy. in mTORC1 activation by inhibiting Sestrin $1 / 2(20,21)$. mTORC1 is stimulated by leucine and glutamine in Rag GTPase-dependent and independent fashion, respectively. Glutamine stimulates mTORC1 in RagA and RagB double knockout cells while existence of v-ATPase (proton pump) is required. Glutamine-mediated stimulation of $\mathrm{mTORC} 1$ requires adenosine diphosphate ribosylation factor (ARF)-1 GTPase for appropriate regulation (22).

Rag family of GTPases is one of the crucial links between amino acids and mTORC1. First group consisting of RagA and RagB binds to GTP while the second group of RagC and RagD has affinity for GDP. Upon nutrient provision, each member of the group can make heterodimer only with a member of another group (RagA-RagC or RagA-RagD; RagB-RagC or RagB-RagD) $(23,24)$. Sestrin $1 / 2$ interacts with GATOR2 to inhibit mTORC1 signaling (Fig. 1) (21, 25, 26). In this context, SLC38A9 is one of the strong candidates for sensing arginine at lysosome (27).

\section{Growth factors}

In higher eukaryotes, cell growth and proliferation rely on long-range communication to coordinate distribution of nutrients $(1,28)$. Phosphatidylinositol 3-kinase (PI 3-kinase)dependent pathway regulates mTORC1 and is affected by insulin. Thus mTORC1 mediates crosstalk between amino acids and insulin signaling (Fig. 1) (29). Communication is mediated by growth factors such as insulin, PDGF, VEGF, EGF, and IGF1 (30). Insulin and IGF, when binding to receptors, activate mTORC1 through PI3K and AKT. Insulin and other growth factors bind RTK stimulating recruitment and activation of PI3K. Phosphatidylinositol-3,4,5-trisphosphate (PIP3) is generated by action of PI3K activity, that activates
AKT (30).

AKT phosphorylates TSC2 and inactivates its complex with TSC1 (31). The active complex has GTPase activity and converts Rheb-bound GTP into GDP and thus inactivates Rheb-mediated activation of mTORC1 (32). ERK inhibits TSC1/2 to relieve activity of mTORC1 activity as well as activates RSK (ribosomal s6 kinase) resulting in phosphorylationmediated increased activity of RAPTOR (30). AKT phosphorylates TSC2 and inactivates its complex formation with TSC1 (31).

\section{Glucose, fatty acid, and energy status}

All cellular processes need energy in the form of ATP. Being a major regulator of growth and proliferation, it is logical that mTORC1 activity must be under the control of energy status of the cell (33). Glycolysis, citric acid cycle, $\beta$-oxidation, and oxidative respiration all lead to conversion of nutrients into ATP (34-36). Upon nutrient scarcity, ATP level of cells quickly fall and AMPK is stimulated (37), subsequently activates and inactivates TSC2 (38) and Raptor (39), respectively. This mechanism provides an AMPK-facilitated pathway for mTORC1 to sense ratio of AMP/ATP.

mTORC1 signaling inhibits fatty acid oxidation. Ketone bodies are produced as a result of acetyl-CoA released from $\beta$-oxidation, that either enter TCA cycle or, under nutrient deficiency into the liver (40). Glucose passes through transporters and after being converted into energy, inhibits AMPK, subsequently reviving mTORC1 $(41,42)$. Fatty acids are transported through specific transporters such as fatty-acid-transport protein (FATP) and fatty-acid-binding protein (FABP) families, eventually entering the citric acid cycle to produce energy and activate mTORC1 (Fig. 1) (43-45). 
Glucose entering cells after passing through glycolysis are subjected to Kreb cycle inside the mitochondria. When energy status of the cell is stabilized, AMPK \& TSC $1 / 2$ are inhibited and mTORC1 activity is revived.

\section{mTORC1 REGULATION BY ITS INTERACTING PARTNERS}

mTORC1 is affected by a plethora of factors such as energy

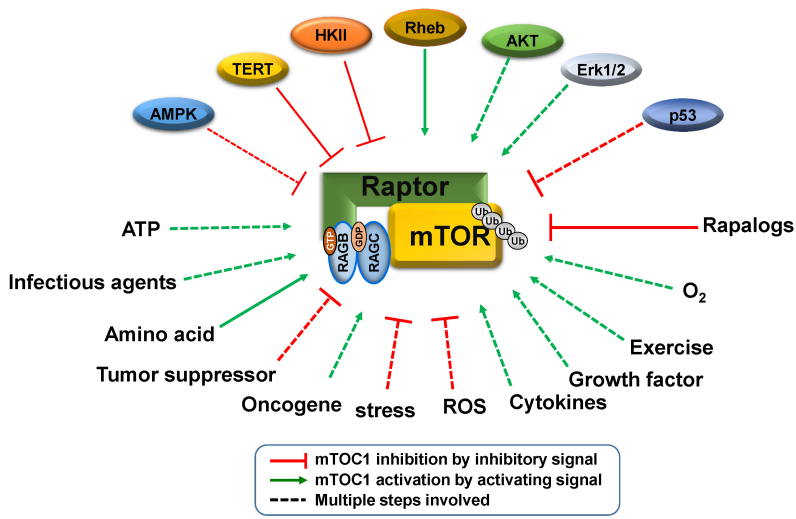

Fig. 2. mTORC1 interacting proteins and external factors that affect activity of this complex. mTORC1 is regulated by a plethora of gene products. AMPK, TERT, HKII, and p53 have inhibitory effect while Rheb, AKT, and ERK activate the effect on this complex. Almost all kinds of stress and nutrients regulate activity of mTORC1. Cellular nutrients, energy status, and growth factors affect mTORC1 activity. status, $\mathrm{O}_{2}$ level, cytokines, $\mathrm{ROS}$ and many more (30). All these factors affect mTORC1 signaling that leads to cellular growth conditions by regulating metabolic processes (Fig. 2).

mTORC1 interacting elements such as p70S6K and 4E-BP1 bind to RPTOR (Regulatory-associated protein of mTOR) through their TOS motif mTOR signaling motif) (46). Immunoprecipitation study revealed that HK-II links to and restricts the autophagy suppressor, mTOR complex 1 (mTORC1), and this binding is promoted in hypoglycemic condition (47). Similarly, TERT restricts mTORC1 under amino acid starvation (11).

mTORC1 suppression by p53 requires TSC1 and TSC2. Formation of TSC1/TSC2 complex is mandatory for p53-dependent mTORC1 inhibition. p53 stimulation has potential to inhibit activity of mTORC1 through a pathway analogous to the withdrawal of energy. This uncommon regulatory pathway is crucial for and contributes to tumor suppressive roles of p53 (48). There can be an alternative approach: TERT has a regulatory (like a rheostat) effect on mTORC1. TERT binds and restricts activated mTORC1 and it is detached from the inactivated complex (11). Some regulatory proteins need to have a TOS consensus sequence $[F(D / E)$ $(\mathrm{F} / \mathrm{I} / \mathrm{L} / \mathrm{M})(\mathrm{D} / \mathrm{E})(\mathrm{L} / \mathrm{l})]$ to bind to Raptor leading to presence in mTORC1 $(46,47)$.

\section{ROLE OF MTORC1 IN DIABETES AND HYPERTRIGLYCERIDEMIA}

Diabetes mellitus (DM) is a heterogeneous metabolic disorder of chronic hyperglycemia (49). Among total diabetic patients, the ratio of patients with insulin dependent diabetes mellitus (Type 1, IDDM) is only $5-10 \%$ with a major cause of the

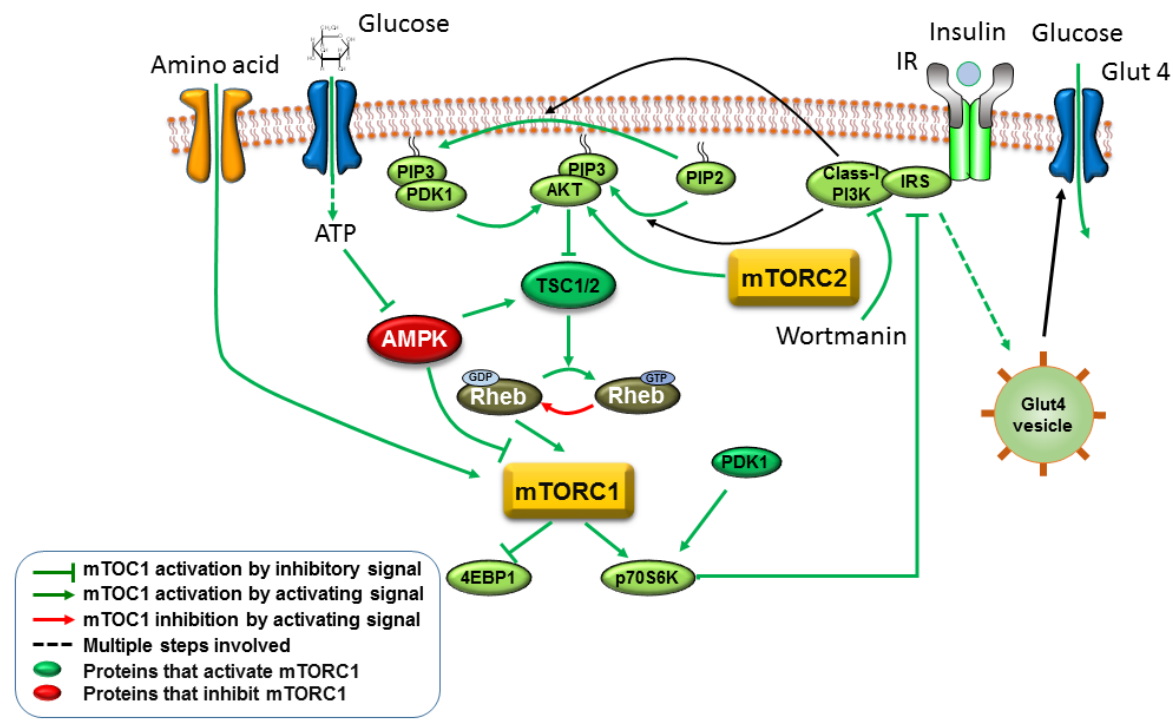

Fig. 3. Insulin resistance by inhibition of IRS under constitutive activation of mTORC1. mTORC1 is activated from high energy diet through uptake of amino acids and glucose. High activity of mTORC1 over a long period inhibits IRS through p70S6K. Thus, IRS loses its capability for transfer of glucose transporters to the cell surface. 
destruction of $\beta$-cells of the pancreas by cell-mediated autoimmune responses (50). Whereas $90-95 \%$ patients are those with $\mathrm{T} 2 \mathrm{D}$ having insulin deprivation or resistance are termed as non-insulin dependent diabetes mellitus (NIDDM) or adult-onset diabetes (50). Rapid increase in T2D prevalence worldwide has been associated with a Western, obesogenic lifestyle (51).

It is important to consider that diabetes is linked to more than one organ and diabetic cardiomyopathy is reported to cause heart attack, a leading cause of morbidity and mortality in diabetic patients. Metabolic profiles of diabetic patients are highly disturbed, having increased level of glucose and lipids, causing hyperglycemia and hyperlipidemia, respectively, as a result of insulin resistance (52-54).

In mammals, the liver is the main organ that controls physiology of the whole body in response to nutrients (55). Hyperactivation of mTORC1 regulates insulin and growth factor signaling through insulin receptor substrates (IRS) (56). mTORC1 has been associated with Type 1 and T2D (56). Class I PI3-Kinases are key components of the insulin signaling pathway $(57,58)$. Prolonged activation of mTORC1, under high energy diet, inhibits IRS through p70S6K (Fig. 3) (59). This pathway renders IRS incapable of transferring glucose transporters onto the cell surface, increasing blood glucose level (60-62). This finally leads to T2D.

Hypertriglyceridemia is triggered by activation of hepatic mTORC1/S6k activation (63). Intake of surplus energy in the form of fat and protein is the root cause of metabolic imbalances and metabolic disorders that promote obesity (64). Amino acids produced from dietary proteins, directly enter in the cytoplasmic circulation contribute to activation of mTORC1-p70S6K pathway through several signaling arrays (13-15). Additionally, other intracellular and extracellular signals, such as growth factors, oxygen $\left(\mathrm{O}_{2}\right)$, tension, and energy levels, induce mTORC1 signalling (65). The factors are shown above (Fig. 2).

Expression of the sodium-coupled neutral amino acid transporter (SNAT2) provokes the mTORC1-p70S6K pathway and increases serum triglycerides (TGs) while reducing adipose lipoprotein lipase (LPL). Similarly, expression of hepatic Rheb (Ras-homolog enriched in brain) or active-S6K produces the same metabolic effects, while expression of dominant-negative-p70S6K inhibits increase of hepatic TG in liver-specific SNAT2-expressing mice. Hypertriglyceridemia and adipose LPL up-regulation are transduced between liver and adipose tissue using a neuronal passage comprising afferent vagal and efferent sympathetic nerves (65). Unsaturated $\mathrm{AA}^{\prime} \mathrm{s}$ stimulate mTORC1 involved in developing insulin resistance and obesity (66). Activation of mTORC1 for a lengthy time promotes insulin resistance and potentially exacerbating obesity triggering lipid deposits (7).

Association of the mTORC1-p70S6K pathway with lipid metabolism is the point of interest that contributes to fatty acid biosynthesis (66). mTORC1 is mandatory for denovo lipid synthesis in murine liver (67). mRNA and protein expression of main gluconeogenic enzymes, in specimens of human liver, revealed that levels of only pyruvate carboxylase protein have strong relation with glycaemia. Pyruvate carboxylase specific antisense oligonucleotide (ASO) does not disturb de novo synthesis of fatty acid, lipolysis, or fatty acid oxidation of liver cells (68). High-fat diet increases endogenous glucose production (69).

Excessive synthesis of very-low-density lipoproteins (VLDL), accompanying greater release of triglyceride \& apolipoprotein B100 (apoB100), is central to excess plasma VLDL-TG levels in insulin-resistant diabetic patients (70). Prolonged hyperinsulinemia predisposes liver for insulin resistance that leads to inability of insulin to trigger an increased signal at insulin receptor substrate-2 (71). Up-regulation of sterol regulatory element-binding protein 1c (SREBP-1c) occurs leading to increased lipid synthesis (71). Thus, hyperinsulinemia may be a pivotal cause of hepatic insulin resistance associated with steatosis. Failure of insulin action on skeletal muscle and the liver leads to hyperglycemia (7).

At least three outcomes are implicated with over nutrition-mediated prolonged hyperactivation of mTORC1. As the first outcome, IRS stops responding to insulin signaling, leading to high blood glucose and consistent production of glucose in liver cells (59). Second, there is increased hyperlipidemia and hypertriglyceridemia, causing insulin resistance and overproduction of hepatic glucose that at later stages are converted into fatty acids and cellular lipids deposits $(72,73)$. Third, liver communicates to brain through vagal nerve and then to white adipose tissues through sympathetic nerves. As a result of this inter-tissue communication, lipoprotein lipases in the blood stream decreases and triglyceride level increases (63).

\section{TELOMERE ATTRITION AND DIABETES}

Telomeres are maintained by a plethora of factors including epigenetic, genetic, environmental, and several unknown events $(74,75)$. Specific diets such as grains, vegetables, and fruits have antioxidant and anti-inflammatory properties and may positively reduce telomere shortening (76-79). Increasing body mass index (BMI), through increased inflammation, has inverse relation with telomere length (80). Telomere length has been decreasing by 7 base pairs per unit increase in BMI $\left(\mathrm{kg} / \mathrm{m}^{2}\right)$. It decreases with increase of C-reactive protein (CRP), revealing that inflammation plays a major role in telomere attrition (81). Several studies have revealed that erosion of the telomerase system is associated with both types of diabetes. Short leukocyte telomere length (LTL) is associated with T1D (82). Increasing of oxidative stress, increases aging and obesity that is directly proportional to telomere attrition in T1D and T2D (83). Chronic hyperglycemia and nutritional overload act together to increase oxidative stress leading to increase in activity of the protein kinase $\mathrm{C}$ pathway. This affects insulin 
signaling, as well as secretes pro-inflammatory cytokines (84).

In brief, we can say that short telomeres implicate with diabetes, and may play an important role in pathogenesis and severity of T2D. Shorter telomeres increase probability of beta-cell senescence, leading to reduced cell mass and decreased insulin level (85). Mice with short telomeres reveal disturbed metabolism through mitochondrial dysfunction (85). In this context, deletion of TERC exhibited a prominent effect. Additionally, telomere shortening may attenuate calciummediated insulin exocytosis (85). Finally, inhibition of p53 activity can reverse cellular senescence of adipocyte and insulin resistance (86).

\section{CHRONIC INHIBITION AND RESTRICTION OF mTORC1 BY TERT AND HKII}

Rapamycin partially protects against insulin resistance, in vivo (87). It was suggested that rapamycin may serve as a promising drug to control hyperactive mTORC1 and insulin resistance in obesity. But prolonged suppression of mTORC1-p70S6K signaling by rapamycin treatment upsets lipid and glucose metabolic rate (88). Chronic intake of rapamycin causes hyperlipidemia and stimulates glucose intolerance (89-91). In conclusion, rapamycin induces a diabetic condition, promoting insulin resistance and dropping $\beta$-cell function and mass $(91,92)$.

TERT binds and restricts mTORC1, but binding decreases when mTORC1 is alternatively inhibited by use of rapamycin $(11,93,94)$. This indicates that TERT predominately binds to the activated form of mTORC1. Recently, mTORC1 inhibiting effect of TERT has been explored $(11,12)$. In this context, we may choose an example of TERT with a regulatory effect on mTORC1. Similarly, the restricting effect of HKII (47) can be used to control hyperactivation of mTORC1. Similarly, HKII binds and inhibits mTORC1 upon glucose starvation where its TOS motif is required for this activity (47). It is important to note that restriction of mTORC1 by TERT and HKII follows opposite nutrient status. It appears that TERT will work under high nutrient status and HKII will be effective under starvation. In addition to TERT and mTORC1, the function of TOS motifs of Deptor and PRAS 40 can be tested for regulatory effect on kinase activity of the complex (Table 1).

We conclude that mTORC1 hyperactivation may lead to insulin resistance, hypertriglyceridemia, and diabetes. Chronic inhibition of mTORC1 exacerbates the pathological condition. Therefore, we propose that hyperactivation of mTORC1 can be controlled by using a specific domain or full length TERT and/or HKII. Further studies are required to identify functional domains of TERT and HKII. This may lead to an effective drug discovery that can regulate mTORC1 activity.

\section{ACKNOWLEDGEMENTS}

The study was supported by HEC, Pakistan and by the

Table 1. Raptor- interacting proteins

\begin{tabular}{|c|c|c|c|c|}
\hline Protein & TOS motif & Bioinformatics & Co-IP with Raptor & Effect on mTORC1 \\
\hline \multicolumn{2}{|c|}{ TOS motif } & \multicolumn{3}{|c|}{$\mathrm{F}(\mathrm{D} / \mathrm{E})(\mathrm{F} / / \mathrm{L} / \mathrm{M})(\mathrm{D} / \mathrm{E})(\mathrm{L} / \mathrm{I})(62)$} \\
\hline Raptor & No & --DLLGRFLDLGPWAV_- ${ }^{\mathrm{a}, \mathrm{b}}$ & NA & Agonist (95) \\
\hline S6K & Yes & -MAGVFDIDLDQPE-- ${ }^{c}$ & Yes (97) & Substrate (60) \\
\hline 4EBP1 & Yes & -EESQFEMDI- - & Yes (97) & Substrate (46) \\
\hline HKII & Yes & -RRGDFDIDIVAVV-- & Yes (62) & Antagonist (47) \\
\hline Sqstm1 & No & -LGIEVDIDVEEHGG-- & Yes (5) & Agonist $(5,6)$ \\
\hline TRAF6 & No & --YDVEFDPPLESKY_- & Yes (6) & Agonist (5) \\
\hline mTOR & No & --LIYVFDVEESRELE-- & Yes (99) & Agonist (99) \\
\hline TERT & No & -SSGLFDVFLRFMC-- & Yes (94) & Antagonist $(11,12)$ \\
\hline Rag A (GTP) & No & --LIYVFDVEESRELE-- & Yes (21) & Agonist $(23,100)$ \\
\hline Rag C (GDP) & No & --PDMNFEVFIHKVD-- & Yes (21) & Agonist $(23,100)$ \\
\hline Rag B (GTP) & No & --NTKTFDVEE $\underline{\text { HSHVR- }}$ & Yes (21) & Agonist $(23,100)$ \\
\hline Rag D (GDP) & No & --TDINFEVFIHKVD-- & Yes (21) & Agonist $(23,100)$ \\
\hline Deptor & No & --GAQQRELERMAEV- & Yes (102) & Antagonist (101) \\
\hline PRAS40 & No & --NGGLFVMDEDDATL- & Yes $(102,103)$ & Antagonist (102) \\
\hline mLST8 & No & -LWCVETGEIKREY-- & Yes (102) & Not yet found (95) \\
\hline Rheb & No & --SIKSFEVIKVIHG-- & Yes (104) & Agonist (103) \\
\hline Hsp90 & No & -RRAPFDLFENRKK-- & Yes (105) & Agonist (104) \\
\hline
\end{tabular}

${ }^{\text {a } T h e ~ a m i n o ~ a c i d ~ d i f f e r i n g ~ f r o m ~ t h e ~ T O S ~ m o t i f ~ a r e ~ u n d e r l i n e d . ~}$

${ }^{b}$ Yellow highlighted motifs differ from TOS motif with respect to one or more amino acids.

${ }^{\mathrm{c}}$ Green highlighted motifs exactly match with TOS motif. 
National Research Foundation funded by the Korean government (NRF-2015R1A2A1A01003845).

\section{CONFLICTS OF INTEREST}

The authors have no conflicting interests.

\section{REFERENCES}

1. Workman JJ, Chen HF and Laribee RN (2014) Environmental signaling through the mechanistic target of rapamycin complex 1 mTORC1 goes nuclear. Cell Cycle 13, 714-725

2. Dalle Pezze P, Sonntag AG, Thien A et al (2012) A dynamic network model of mTOR signaling reveals TSC-independent mTORC2 regulation. Sci Signal 5, ra25

3. Cybulski $N$ and Hall MN (2009) TOR complex 2: a signaling pathway of its own. Trends in Biochemical Sciences 34, 620-627

4. Kim DH, Sarbassov DD, Ali SM et al (2002) mTOR interacts with raptor to form a nutrient-sensitive complex that signals to the cell growth machinery. Cell 110, 163-175

5. Linares JF, Duran A, Yajima T, Pasparakis M, Moscat J and Diaz-Meco MT (2013) K63 polyubiquitination and activation of mTOR by the p62-TRAF6 complex in nutrient-activated cells. Mol Cell 51, 283-296

6. Duran A, Amanchy R, Linares JF et al (2011) p62 is a key regulator of nutrient sensing in the mTORC1 pathway. Mol Cell 44, 134-146

7. Laplante M and Sabatini DM (2012) mTOR signaling in growth control and disease. Cell 149, 274-293

8. Laplante M and Sabatini DM (2009) mTOR signaling at a glance. J Cell Sci 122, 3589-3594

9. Avila-Flores A, Santos T, Rincon E and Merida I (2005) Modulation of the mammalian target of rapamycin pathway by diacylglycerol kinase-produced phosphatidic acid. J Biol Chem 280, 10091-10099

10. Zoncu R, Efeyan A and Sabatini DM (2011) mTOR: from growth signal integration to cancer, diabetes and ageing. Nat Rev Mol Cell Biol 12, 21-35

11. Ali M, Devkota S, Roh JI, Lee J and Lee HW (2016) Telomerase reverse transcriptase induces basal and amino acid starvation-induced autophagy through mTORC1. Biochem Biophys Res Commun 478, 1198-1204

12. Cheng $\mathrm{H}$, Fan $\mathrm{X}$, Lawson WE, Paueksakon $\mathrm{P}$ and Harris RC (2015) Telomerase deficiency delays renal recovery in mice after ischemia-reperfusion injury by impairing autophagy. Kidney Int 88, 85-94

13. Um SH, D'Alessio D and Thomas G (2006) Nutrient overload, insulin resistance, and ribosomal protein S6 kinase 1, S6K1. Cell Metab 3, 393-402

14. Taylor PM (2014) Role of amino acid transporters in amino acid sensing. Am J Clin Nutr 99, 223S-230S

15. Jewell JL, Russell RC and Guan K-L (2013) Amino acid signalling upstream of mTOR. Nat Rev Mol Cell Biol 14, 133-139

16. Jia GH, Aroor AR, Martinez-Lemus LA and Sowers JR
(2014) Overnutrition, mTOR signaling, and cardiovascular diseases. Am J Physiol Regul Integr Comp Physiol 307, R1198-R1206

17. Greider CW (2016) Regulating telomere length from the inside out: the replication fork model. Gene Dev 30, 1483-1491

18. Sahin E, Colla S, Liesa M et al (2011) Telomere dysfunction induces metabolic and mitochondrial compromise. Nature 470, 359

19. Kuhlow D, Florian S, von Figura G et al (2010) Telomerase deficiency impairs glucose metabolism and insulin secretion. Aging (Albany NY) 2, 650

20. Lynch CJ (2001) Role of leucine in the regulation of mTOR by amino acids: Revelations from structure-activity studies. J Nutr 131, 861s-865s

21. Wolfson RL, Chantranupong L, Saxton RA et al (2016) Sestrin2 is a leucine sensor for the mTORC1 pathway. Science (New York, N.Y.) 351, 43-48

22. Jewell JL, Kim YC, Russell RC et al (2015) Differential regulation of mTORC1 by leucine and glutamine. Science 347, 194-198

23. Sancak Y, Peterson TR, Shaul YD et al (2008) The Rag GTPases bind raptor and mediate amino acid signaling to mTORC1. Science 320, 1496-1501

24. Kim E, Goraksha-Hicks P, Li L, Neufeld TP and Guan KL (2008) Regulation of TORC1 by Rag GTPases in nutrient response. Nat Cell Biol 10, 935-945

25. Chantranupong L, Wolfson RL, Orozco JM et al (2014) The Sestrins interact with GATOR2 to negatively regulate the amino-acid-sensing pathway upstream of mTORC1. Cell Rep 9, 1-8

26. Parmigiani A, Nourbakhsh A, Ding B et al (2014) Sestrins inhibit mTORC1 kinase activation through the GATOR complex. Cell Rep 9, 1281-1291

27. Wang S, Tsun Z-Y, Wolfson RL et al (2015) Lysosomal amino acid transporter SLC38A9 signals arginine sufficiency to mTORC1. Science 347, 188-194

28. Floyd S, Favre C, Lasorsa FM et al (2007) The insulin-like growth Factor-I-mTOR signaling pathway induces the mitochondrial pyrimidine nucleotide carrier to promote cell growth. Mol Biol Cell 18, 3545-3555

29. Hinault C, Mothe-Satney I, Gautier N, Lawrence JC Jr, and Van Obberghen E (2004) Amino acids and leucine allow insulin activation of the $\mathrm{PKB} / \mathrm{mTOR}$ pathway in normal adipocytes treated with wortmannin and in adipocytes from db/db mice. FASEB J 18, 1894-1896

30. Dibble CC and Manning BD (2013) Signal integration by mTORC1 coordinates nutrient input with biosynthetic output. Nat Cell Biol 15, 555-564

31. Inoki K, Li Y, Zhu TQ, Wu J and Guan KL (2002) TSC2 is phosphorylated and inhibited by Akt and suppresses mTOR signalling. Nat Cell Biol 4, 648-657

32. Inoki K, Li Y, Xu T and Guan KL (2003) Rheb GTPase is a direct target of TSC2 GAP activity and regulates mTOR signaling. Gene Dev 17, 1829-1834

33. Catania C, Binder E and Cota D (2011) mTORC1 signaling in energy balance and metabolic disease. Int J Obes (Lond.) 35, 751-761

34. Nakashima RA, Paggi MG and Pedersen PL (1984) Contributions of glycolysis and oxidative phosphorylation 
to adenosine $5^{\prime}$-triphosphate production in AS-30D hepatoma cells. Cancer Res 44, 5702-5706

35. Vander Heiden MG, Cantley LC and Thompson CB (2009) Understanding the Warburg effect: the metabolic requirements of cell proliferation. Science 324, 1029-1033

36. Bartlett K and Eaton S (2004) Mitochondrial $\beta$-oxidation. Eur J Biochem 271, 462-469

37. Sato R, Goldstein JL and Brown MS (1993) Replacement of serine-871 of hamster 3-hydroxy-3-methylglutaryl-coa reductase prevents phosphorylation by AMP-activated kinase and blocks inhibition of sterol synthesis induced by ATP depletion. Proc Natl Acad Sci U S A 90, 9261-9265

38. Inoki K, Zhu TQ and Guan KL (2003) TSC2 mediates cellular energy response to control cell growth and survival. Cell 115, 577-590

39. Gwinn DM, Shackelford DB, Egan DF et al (2008) AMPK phosphorylation of raptor mediates a metabolic checkpoint. Mol Cell 30, 214-226

40. Sengupta S, Peterson TR, Laplante M, Oh S and Sabatini DM (2010) mTORC1 controls fasting-induced ketogenesis and its modulation by ageing. Nature 468, 1100-1104

41. Zielinski DC, Jamshidi N, Corbett AJ, Bordbar A, Thomas A and Palsson BO (2017) Systems biology analysis of drivers underlying hallmarks of cancer cell metabolism. Sci Rep 7, 41241

42. Towler MC and Hardie DG (2007) AMP-activated protein kinase in metabolic control and insulin signaling. Circ Res 100, 328-341

43. Kim J, Kundu M, Viollet B and Guan KL (2011) AMPK and $\mathrm{mTOR}$ regulate autophagy through direct phosphorylation of Ulk1. Nat Cell Biol 13, 132-141

44. Hardwick JP, Eckman K, Lee YK et al (2013) Eicosanoids in metabolic syndrome. Adv Pharmacol (San Diego, Calif.) $66,157-266$

45. Kerner J and Hoppel C (2000) Fatty acid import into mitochondria. Biochim Biophys Acta 1486, 1-17

46. Nojima H, Tokunaga C, Eguchi $\mathrm{S}$ et al (2003) The mammalian target of rapamycin (mTOR) partner, raptor, binds the mTOR substrates, p70 S6 kinase and 4E-BP1, through their TOR signaling (TOS) motif. J Biol Chem 278, 15461-15464

47. Roberts DJ, Tan-Sah VP, Ding EY, Smith JM and Miyamoto S (2014) Hexokinase-Il positively regulates glucose starvation-induced autophagy through TORC1 inhibition. Mol Cell 53, 521-533

48. Feng $Z$, Zhang $H$, Levine AJ and Jin S (2005) The coordinate regulation of the p53 and mTOR pathways in cells. Proc Natl Acad Sci U S A 102, 8204-8209

49. Bukhari SA, Javed S, Ali M, Shahzadi A and Rehman M (2015) Serum haematological and biochemical indices of oxidative stress and their relationship with DNA damage and homocysteine in Pakistani type II diabetic patients. Pak J Pharm Sci 28, 881-889

50. Association AD (2010) Diagnosis and classification of diabetes mellitus. Diabetes care 33, S62-S69

51. Billington CJ, Epstein LH, Goodwin NJ et al (2000) Overweight, obesity, and health risk. Arch Intern Med 160, 898-904

52. Völkers M, Doroudgar S, Nguyen N et al (2014) PRAS40 prevents development of diabetic cardiomyopathy and improves hepatic insulin sensitivity in obesity. Embo Mol Med 6, 57-65

53. Samuel VT and Shulman Gl (2012) Mechanisms for insulin resistance: common threads and missing links. Cell 148, 852-871

54. Han J, Li E, Chen L et al (2015) The CREB coactivator CRTC2 controls hepatic lipid metabolism by regulating SREBP1. Nature 524, 243-246

55. Cornu M, Oppliger W, Albert V et al (2014) Hepatic mTORC1 controls locomotor activity, body temperature, and lipid metabolism through FGF21. Proc Natl Acad Sci U S A 111, 11592-11599

56. Passtoors WM, Beekman M, Deelen J et al (2013) Gene expression analysis of mTOR pathway: association with human longevity. Aging Cell 12, 24-31

57. Engelman JA, Luo J and Cantley LC (2006) The evolution of phosphatidylinositol 3-kinases as regulators of growth and metabolism. Nat Rev Genet 7, 606-619

58. Chalhoub N and Baker SJ (2009) PTEN and the PI3-kinase pathway in cancer. Annu Rev Pathol 4, 127-150

59. Um SH, Frigerio F, Watanabe $M$ et al (2004) Absence of S6K1 protects against age- and diet-induced obesity while enhancing insulin sensitivity. Nature 431, 200-205

60. Thedieck K, Holzwarth B, Prentzell MT et al (2013) Inhibition of mTORC1 by astrin and stress granules prevents apoptosis in cancer cells. Cell 154, 859-874

61. Melnik BC, John SM, Carrera-Bastos $P$ and Cordain L (2012) The impact of cow's milk-mediated mTORC1signaling in the initiation and progression of prostate cancer. Nutr Metab 9, 74

62. Chang L, Chiang S-H and Saltiel AR (2004) Insulin signaling and the regulation of glucose transport. Mol Med 10, 65-71

63. Uno K, Yamada T, Ishigaki $Y$ et al (2015) A hepatic amino acid/mTOR/S6K-dependent signalling pathway modulates systemic lipid metabolism via neuronal signals. Nat Commun 6, 7940

64. Tremblay F, Lavigne C, Jacques H and Marette A (2007) Role of dietary proteins and amino acids in the pathogenesis of insulin resistance. Annu Rev Nutr 27, 293-310

65. Zoncu R, Efeyan A and Sabatini DM (2011) mTOR: from growth signal integration to cancer, diabetes and ageing. Nat Rev Mol Cell Biol 12, 21-35

66. Laplante M and Sabatini DM (2010) mTORC1 activates SREBP-1C and uncouples lipogenesis from gluconeogenesis. Proc Natl Acad Sci U S A 107, 3281-3282

67. Yecies JL, Zhang HH, Menon S et al (2011) Akt stimulates hepatic SREBP1c and lipogenesis through parallel mTORC1-dependent and independent pathways. Cell Metab 14, 21-32

68. Kumashiro N, Beddow SA, Vatner DF et al (2013) Targeting pyruvate carboxylase reduces gluconeogenesis and adiposity and improves insulin resistance. Diabetes 62, 2183-2194

69. Song SM, Andrikopoulos S, Filippis C, Thorburn AW, Khan D and Proietto J (2001) Mechanism of fat-induced hepatic gluconeogenesis: effect of metformin. Am J 
Physiol-Endoc M 281, E275-E282

70. Ginsberg HN (2000) Insulin resistance and cardiovascular disease. J Clin Invest 106, 453-458

71. Shimomura I, Matsuda M, Hammer RE, Bashmakov Y, Brown MS and Goldstein JL (2000) Decreased IRS-2 and increased SREBP-1c lead to mixed insulin resistance and sensitivity in livers of lipodystrophic and ob/ob mice. Mol Cell 6, 77-86

72. Jin ES, Szuszkiewicz-Garcia M, Browning JD, Baxter JD, Abate $N$ and Malloy CR (2015) Influence of liver triglycerides on suppression of glucose production by insulin in men. J Clin Endocr Metab 100, 235-243

73. Seppala-Lindroos A, Vehkavaara S, Hakkinen AM et al (2002) Fat accumulation in the liver is associated with defects in insulin suppression of glucose production and serum free fatty acids independent of obesity in normal men. J Clin Endocr Metab 87, 3023-3028

74. Munoz P, Blanco R, Flores JM and Blasco MA (2005) XPF nuclease-dependent telomere loss and increased DNA damage in mice overexpressing TRF2 result in premature aging and cancer. Nat Genet 37, 1063-1071

75. Steinert S, Shay JW and Wright WE (2004) Modification of subtelomeric DNA. Mol Cell Biol 24, 4571-4580

76. Nettleton JA, Diez-Roux A, Jenny NS, Fitzpatrick AL and Jacobs DR (2008) Dietary patterns, food groups, and telomere length in the Multi-Ethnic Study of Atherosclerosis (MESA). Am J Clin Nutr 88, 1405-1412

77. Mirabello L, Huang WY, Wong JY et al (2009) The association between leukocyte telomere length and cigarette smoking, dietary and physical variables, and risk of prostate cancer. Aging Cell 8, 405-413

78. Knoops KT, de Groot LC, Kromhout D et al (2004) Mediterranean diet, lifestyle factors, and 10-year mortality in elderly European men and women: the HALE project. JAMA 292, 1433-1439

79. Ornish D, Lin J, Daubenmier J et al (2008) Increased telomerase activity and comprehensive lifestyle changes: a pilot study. Lancet Oncol 9, 1048-1057

80. von Zglinicki T (2002) Oxidative stress shortens telomeres. Trends Biochem Sci 27, 339-344

81. Rode L, Nordestgaard BG, Weischer M and Bojesen SE (2014) Increased body mass index, elevated C-reactive protein, and short telomere length. J Clin Endocrinol Metab 99, E1671-E1675

82. Jeanclos E, Krolewski A, Skurnick J et al (1998) Shortened telomere length in white blood cells of patients with IDDM. Diabetes 47, 482-486

83. Ma D, Zhu W, Hu S, Yu X and Yang Y (2013) Association between oxidative stress and telomere length in Type 1 and Type 2 diabetic patients. J Endocrinol Invest 36, 1032-1037

84. Geraldes P and King GL (2010) Activation of protein kinase $\mathrm{C}$ isoforms and its impact on diabetic complications. Circ Res 106, 1319-1331

85. Guo N, Parry EM, Li L-S et al (2011) Short telomeres compromise $\beta$-cell signaling and survival. PLoS One 6, e17858

86. Minamino T, Orimo M, Shimizu I et al (2009) A crucial role for adipose tissue p53 in the regulation of insulin resistance. Nat Med 15, 1082-1087
87. Berg CE, Lavan BE and Rondinone CM (2002) Rapamycin partially prevents insulin resistance induced by chronic insulin treatment. Biophy Res Commun 293, 1021-1027

88. Stallone G, Infante B, Grandaliano G and Gesualdo L (2009) Management of side effects of sirolimus therapy. Transplantation 87, S23-26

89. Cruzado JM (2008) Nonimmunosuppressive effects of mammalian target of rapamycin inhibitors. Transplant Rev 22, 73-81

90. Morrisett JD, Abdel-Fattah G, Hoogeveen R et al (2002) Effects of sirolimus on plasma lipids, lipoprotein levels, and fatty acid metabolism in renal transplant patients. J Lipid Res 43, 1170-1180

91. Houde VP, Brûlé S, Festuccia WT et al (2010) Chronic rapamycin treatment causes glucose intolerance and hyperlipidemia by upregulating hepatic gluconeogenesis and impairing lipid deposition in adipose tissue. Diabetes 59, 1338-1348

92. Fraenkel $M$, Ketzinel-Gilad $M$, Ariav $Y$ et al (2008) mTOR inhibition by rapamycin prevents $\beta$-cell adaptation to hyperglycemia and exacerbates the metabolic state in type 2 diabetes. Diabetes 57, 945-957

93. Sundin T, Peffley DM and Hentosh P (2013) Disruption of an hTERT-mTOR-RAPTOR protein complex by a phytochemical perillyl alcohol and rapamycin. Mol Cell Biochem 375, 97-104

94. Kawauchi K, Ihjima K and Yamada O (2005) IL-2 increases human telomerase reverse transcriptase activity transcriptionally and posttranslationally through phosphatidylinositol 3'-kinase/Akt, heat shock protein 90, and mammalian target of rapamycin in transformed NK cells. J Immunol 174, 5261-5269

95. Guertin DA, Stevens DM, Thoreen CC et al (2006) Ablation in mice of the mTORC components raptor, rictor, or mLST8 reveals that $\mathrm{mTORC} 2$ is required for signaling to Akt-FOXO and PKC alpha but not S6K1. Dev Cell 11, 859-871

96. Schalm SS and Blenis J (2002) Identification of a conserved motif required for mTOR signaling. Curr Biol $12,632-639$

97. Linares JF, Duran A, Yajima T, Pasparakis M, Moscat J and Diaz-Meco MT (2013) K63 polyubiquitination and activation of mTOR by the p62-TRAF6 complex in nutrient-activated cells. Mol Cell 51, 283-296

98. Rosner M, Siegel N, Valli A, Fuchs C and Hengstschlager M (2010) mTOR phosphorylated at S2448 binds to raptor and rictor. Amino Acids 38, 223-228

99. Wrighton KH (2013) Cell signalling: Where the mTOR action is. Nat Rev Mol Cell Biol 14, 191

100. Shimobayashi M and Hall MN (2014) Making new contacts: the mTOR network in metabolism and signalling crosstalk. Nat Rev Mol Cell Biol 15, 155-162

101. Peterson TR, Laplante $M$, Thoreen CC et al (2009) DEPTOR is an mTOR inhibitor frequently overexpressed in multiple myeloma cells and required for their survival. Cell 137, 873-886

102. Sancak Y, Thoreen CC, Peterson TR et al (2007) PRAS40 is an insulin-regulated inhibitor of the $\mathrm{mTORC} 1$ protein kinase. Mol Cell 25, 903-915

103. Long X, Lin Y, Ortiz-Vega S, Yonezawa K and Avruch J 
Restriction of hyperactive mTORC1 in diabetes Muhammad Ali, et al.

(2005) Rheb binds and regulates the mTOR kinase. Curr Biol 15, 702-713

104. Ohji G, Hidayat S, Nakashima A et al (2006) Suppression of the mTOR-raptor signaling pathway by the inhibitor of heat shock protein 90 geldanamycin. J Biochem 139, 129-135 